\title{
Stereotactic Body Radiotherapy Using Cyberknife for Localized Prostate Cancer
}

\author{
Hunjung Kim, ${ }^{1,}{ }^{*}$ Hoonjung Phak, ${ }^{1}$ and Woochul Kim ${ }^{1}$ \\ ${ }^{1}$ Department of Radiation Oncology, Inha University Hospital, Inha University of Medicine, Inchon, Korea \\ "Corresponding author: Hunjung Kim, Department of Radiation Oncology, Inha University Hospital, Inha University of Medicine, Inchon, Korea. Tel: +82-32890-3070, Fax: \\ +82-328903082, E-mail: cancerovercome@gmail.com
}

Received 2015 July 18; Accepted 2015 September 11.

\begin{abstract}
Background: Stereotactic body radiation therapy (SBRT) using Cyberknife delivers high-dose fraction of radiation without increasing toxicity.

Objectives: We present the follow-up outcomes and toxicities of patients with localized prostate cancer treated by use of Cyberknife as a monotherapy.

Patients and Methods: This study was based on a retrospective analysis of the 34 patients treated with SBRT using Cyberknife for localized prostate cancer (26.5\% low risk, 67.6\% intermediate risk, and 5.9\% high risk). Total dose of 36.25 Gy in 5 fractions of 7.25Gy were administered without use of androgen deprivation therapy (ADT). The acute and late toxicities were recorded using the radiation therapy oncology group scale. Prostate-specific antigen (PSA) response was monitored.

Results: Thirty-four patients with a median 52 months (range 12 - 71 month) follow-up were analyzed. The biochemical relapse-free survival was $93.3 \%$. PSA fell to a median of $0.39 \mathrm{ng} / \mathrm{mL}$ at 4 years and PSA bounce occurred in $28.1 \%$ of patients. Acute side effects resolved within 1-2 months of treatment completion. There was no grade 3 and 4 late toxicity observed.

Conclusions: In this study, SBRT using Cyberknife without ADT has provided promising outcomes in localized prostate cancer with good PSA response and minimal toxicity. Hypofractionated SBRT using Cyberknife leads to long-term favorable 5-year biochemical relapse-free survival and minimal toxicity in localized prostate cancer as a monotherapy.
\end{abstract}

Keywords: Prostate Cancer, Stereotactic Body Radiotherapy, Cyberknife

\section{Background}

Prostate cancer is the most common cancer and the second leading cause of death from cancer among men in the United States (1). As an alternative to surgery, various radiation treatment techniques have been developed. The use of stereotactic body radiation therapy(SBRT) has recently emerged as a technique to deliver hypofractionated radiation therapy to the prostate (2-5). The alpha/beta $(\alpha \mid \beta)$ ratio of prostate cancer has been thought to be around 1.5 Gy and lower than the surrounding normal tissue $(6,7)$. Therefore, the use of a hypofractionated dose scheme should lead to a more advantageous therapeutic ratio $(8,9)$. Treatment with high dose-rate (HDR) brachytherapy is well established as a hypofractionated radiation therapy (10). Advanced techniques using SBRT allows high doses of radiation to be delivered precisely to the target tissue while sparing the surrounding healthy tissue, thus achieving high biochemical control and low toxicity (2-4). SBRT using Cyberknife (Accuray, Sunnyvale, CA, USA) delivers hypofractionated treatment regimens with using real-time image guidance to account for intrafraction pro- static motion. Several recent Cyberknife publications report promising clinical efficacy with minimal toxicity (2, 11).

\section{Objectives}

In this report, we present the follow-up outcomes and toxicities of patients with localized prostate cancer treated by use of Cyberknife as a monotherapy.

\section{Patients and Methods}

\subsection{Patient Characteristics}

A prospective protocol-based study for the treatment of localized prostate cancer with CyberKnife robotic radiosurgery system began from March 2008 at Inha university hospital in Inchon. Since then, thirty-four patients have been treated (Table 1). Eligible patients had newly diagnosed, biopsy-proven localized prostate cancer. Exclusion criteria included clinical stage T3, involved lymph nodes or 
distant metastases on imaging and/or prior pelvic radiotherapy. The study was approved by the ethical committee for clinical trials of our institution and the retrospective data was prospectively collected in our institutional database.

\subsection{SBRT Treatment Planning and Delivery}

Four or more gold fiducial markers were implanted transperineally into the prostate. After seven days, patients underwent MR imaging and thin-cut CT scan. Fused CT and MR images were used for the treatment planning. The clinical target volume (CTV) included the prostate and proximal seminal vesicles. The planning target volume (PTV) equaled the CTV expanded $3 \mathrm{~mm}$ posteriorly and $5 \mathrm{~mm}$ in all other dimensions. The prescription dose was 36.25 Gy, delivered in five fractions, was prescribed to the PTV. The prescription dose covered at least $95 \%$ of the planning target volume, normalized to the 75 - $85 \%$ isodose line (mean homogeneity index of 1.28 [range, $1.24-1.43$ ]). The rectal dose-volume goals were $<50 \%$ of the rectal volume receiving $50 \%$ of the prescribed dose, $<20 \%$ receiving $80 \%$ of the dose, $<10 \%$ receiving $90 \%$ dose, and $<5 \%$ receiving $100 \%$ of the dose. Treatments were given over 5 consecutive days. Androgen deprivation therapy(ADT) was not applied to anyone.

\subsection{Follow-up and Toxicity Scoring}

Patients were followed every 3 months during the first year and every 6 - 12 months thereafter. Prostate-specific antigen (PSA) levels were obtained at each follow-up. Biochemical failure was defined as an increase of at least $2 \mathrm{ng} / \mathrm{mL}$ from the nadir PSA according to the Phoenix definition (12). We calculate the decline velocity of PSA over an interval of time from the completion of radiotherapy to 1, 2,3 and 4 years following treatment. The velocity $(\mathrm{ng} / \mathrm{mL} / \mathrm{year})$ was calculated as the regression coefficient in a linear regression model for each individual. PSA values taken after the start of ADT were excluded. PSA bounce was defined as an absolute increase of $0.2 \mathrm{ng} / \mathrm{mL}$ from the previous PSA level, followed by a subsequent decrease (13). The t test was performed to compare mean values and ANOV in continuous variables. Statistical analysis was performed using the IMB SPSS software, version 19.0 (SPSS, Inc., IBM, Chicago, IL, USA). Toxicity was documented at follow-up visits using the radiation therapy oncology groups scale.

\section{Results}

All patients completed the treatment. Thirty-four patients with a median 52 month (range 12 - 71 month) followup were analyzed (Table 1 ). The mean age was 68.3 years ( 56 - 77 years).

\subsection{PSA Changes and Biochemical Relapse}

The median pretreatment serum PSA of $7.62 \mathrm{ng} / \mathrm{mL}$ (range $3.45-14.90 \mathrm{ng} / \mathrm{mL}$ ) declined to a median of 0.39 $\mathrm{ng} / \mathrm{mL}$ (range $<0.04-1.62 \mathrm{ng} / \mathrm{mL}$ ) at four years posttreatment(Figure 1). The velocity of PSA declining was maximal in the first year (median $-4.184 \mathrm{mg} / \mathrm{mL} / \mathrm{year}$ ), then velocity of PSA declining was gradually falling off with median values of $-2.084,-1.548$ and $1.032 \mathrm{ng} / \mathrm{mL} / \mathrm{month}$ for duration of 2, 3 and 40 years after Cyberknife, respectively. The median PSA nadir was $0.31 \mathrm{ng} / \mathrm{mL}$ (range, 0.04 - 1.02 $\mathrm{ng} / \mathrm{mL}$ ) with median 33 months. Benign PSA bounces occurred in nine patients (28.1\%) with a median PSA bounce of $0.29 \mathrm{ng} / \mathrm{mL}$ (range, $0.22-1.36 \mathrm{ng} / \mathrm{mL}$ ) and the median time following treatment to the PSA bounce was 10.5 months (range, 6-12 months). There was one biochemical failure, occurring in a high risk patient. Prostate biopsy confirmed local recurrence and ADT was initiated. The five-year actuarial biochemical relapse free survival was 93.3\% (Figure 2). Univariate analysis revealed no statistical significance for biochemical failure for the following prognostic factors: age, NCCN risk groups, Gleason score, T stage, pretreatment PSA.

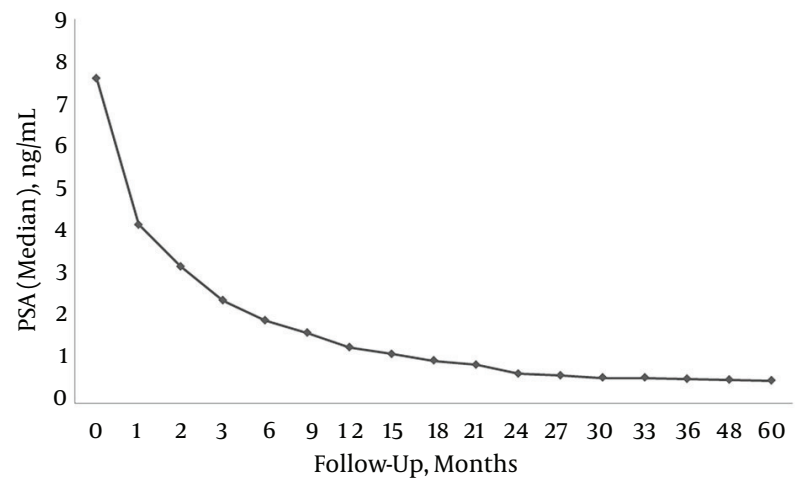

Figure 1. Prostate-Specific Antigen Changes After Stereotactic Body Radiation Therapy

\subsection{Toxicity}

The prevalent acute complain were urinary frequency and rectal pain, usually during the first and second weeks after treatment. Acute grade 2 genitourinary (GU) toxicities were seen in $17.6 \%(n=6)$ and acute 2 gastrointestinal (GI) toxicities in $23.5 \%(n=8)$ (Table 2$)$. No grade 3 or 4 acute GU and GI toxicities occurred. Acute toxicity was usually resolved within 1 - 2 month on basic symptomatic therapy. Late toxicity rate were acceptable without grade 3 and 4 late toxicity. Late grade $2 \mathrm{GU}$ toxicities were observed in $5.9 \%(n=2)$ and grade 2 GI toxicities in $8.8 \%(n=3)$. 
Table 1. Patient and Tumor Characteristics $(n=34)^{\mathrm{a}}$

\begin{tabular}{|c|c|}
\hline Variables & Value \\
\hline Mean age, $y$ & $68.3(56-77)$ \\
\hline \multicolumn{2}{|l|}{ ECOG } \\
\hline 0 & $23(67.6)$ \\
\hline 1 & $11(32.4)$ \\
\hline \multicolumn{2}{|l|}{ T stage } \\
\hline T1c & $2(5.9)$ \\
\hline T2a-b & $14(41.2)$ \\
\hline $\mathrm{T} 2 \mathrm{c}$ & $18(52.9)$ \\
\hline \multicolumn{2}{|l|}{ Gleason score } \\
\hline$\leq 6$ & $14(41.2)$ \\
\hline 7 & $18(52.9)$ \\
\hline$>7$ & $2(5.9)$ \\
\hline Pretreatment PSA $(\mathrm{ng} / \mathrm{mL})$ & $7.62(3.45-14.90)$ \\
\hline \multicolumn{2}{|l|}{ NCCN risk group } \\
\hline Low & $9(26.5)$ \\
\hline \multicolumn{2}{|l|}{ Intermediate } \\
\hline High & $2(5.9)$ \\
\hline \multicolumn{2}{|l|}{ Overall treatment days } \\
\hline 5 days & $20(58.8)$ \\
\hline$>5$ days & $14(52.9)$ \\
\hline
\end{tabular}

Abbreviation: NCCN, national comprehensive cancer network.

${ }^{\mathrm{a}}$ Values are expressed as No. (\%) unless otherwise indicated.

Table 2. Toxicity $^{\mathrm{a}}$

\begin{tabular}{|c|c|c|c|c|}
\hline \multirow[t]{2}{*}{ Toxicity } & \multicolumn{4}{|c|}{ Grade } \\
\hline & I & II & III & IV \\
\hline \multicolumn{5}{|l|}{ Acute } \\
\hline Urinary & 35.3 & 17.6 & & \\
\hline Rectal & 26.4 & 23.5 & & \\
\hline \multicolumn{5}{|l|}{ Late } \\
\hline Urinary & 8.8 & 5.9 & & \\
\hline Rectal & 11.8 & 8.8 & 2.9 & \\
\hline
\end{tabular}

${ }^{\mathrm{a}}$ Values are expressed as \%.

\section{Discussion}

In this report, with long-term follow-up, demonstrates that SBRT using Cyberknife can achieve excellent biochemical control rates and low levels of bladder and rectal toxicity. The rapid decline of PSA level occurred in the first year and PSA fell steadily to achieve very low levels (mean of $0.55 \mathrm{mg} / \mathrm{mL}$ ) within 4 years. Anwar et al. (14) compared the PSA slope between the hypofractionated SBRT and conventionally fractionated external beam radiation therapy (EBRT) for localized prostate cancer and reported that the PSA slope for SBRT was greater than conventionally fractionated EBRT at 2 and 3 years and PSA nadir was significantly lower for SBRT. 


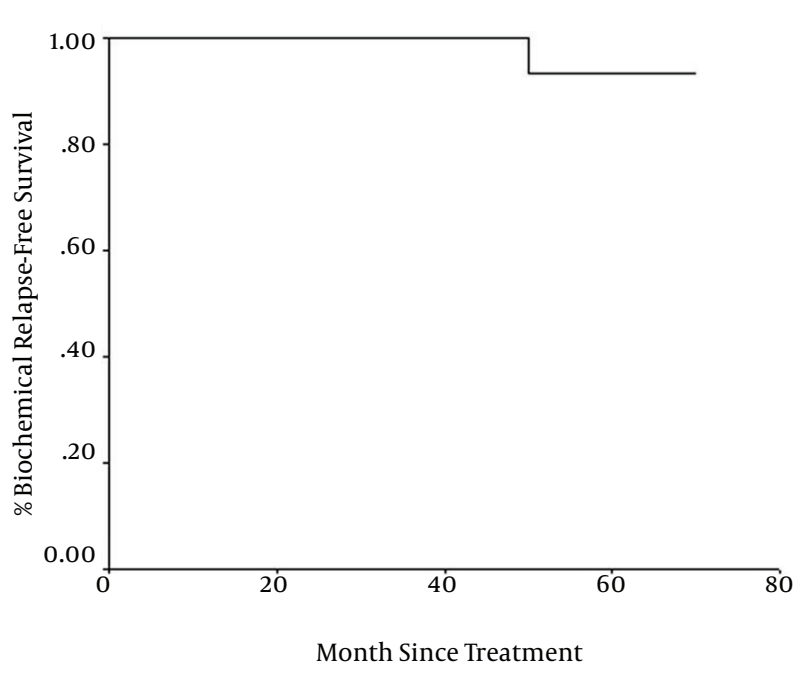

Figure 2. Biochemical Failure-Free Survival Rate After Stereotactic Body Radiation Therapy

Katz et al. (2) demonstrated that PSA decline steadily after treatment and achieve very low mean levels of 0.25 $\mathrm{ng} / \mathrm{mL}$ within 4 - 5 years. In this study, PSA declined rapidly first year and velocity of decline was gradually falling off with follow up times and low PSA nadir of $0.31 \mathrm{mg} / \mathrm{mL}$. These findings support the predictions of estimated $\alpha / \beta$ ratio of $1.5 \mathrm{~Gy}$. Using the linear-quadratic radiobiologic model, 36.25 Gy of Cyberknife yields an equivalent dose (EQD) at $2 \mathrm{~Gy}$ fraction of $91 \mathrm{~Gy}$ for this $\alpha / \beta$ ratio (15).

Recent reports recommended that hypofractionated schedule may provide similar excellent control as other radiation modalities. Arcangeli et al. (16) published a report comparing 80 Gy (2 Gy/fraction) versus 62 Gy (3.1 Gy/fraction) and showed that the hypofractionated schedule is superior to the conventional fractionation in terms of freedom from biochemical failure rate with equivalent toxicity. This is also confirmed by studies of high dose rate brachytherapy (HDR BT) (17-19). Our outcomes are consistent with those that have resulted from HDR BT. Demanes et al. (19) reported the 8 year biochemical control of $97 \%$ in low and intermediate risk prostate patients. However, due to its invasive nature and technical difficulties, use of brachytherapy is less common. Cyberknife allows the delivery of large fractions dose such as HDR BT with submillimeter accuracy to the target with excellent sparing of normal tissue. But there is still a matter of debate about the efficacy and toxicity of hypofractionation with Cyberknife.

Toxicity following SBRT was similar to that following EBRT or brachytherapy. Zelefsky et al. (20) reported result on late toxicity using 81 Gy dose with IMRT in conventional fractionation. The 8 -year actuarial likelihood of grade 2 GI toxicity was $1.6 \%$ and $0.1 \%$ of patients experienced grade 3 rectal toxicity. The 8 -year likelihood of late grade 2 and $3 \mathrm{GU}$ toxicities were $9 \%$ and 3\%, respectively. Katz et al. reported that, among 477 patients with lowand intermediate-risk prostate cancer treated using $\mathrm{Cy}$ berknife, only $1.7 \%$ of grade 3 late toxicity event occurred in patients who received 36.25 Gy in 5 fractions (21). Our current study shows the similar proportion of toxicity.

In this study, PSA bounce was seen in $28.1 \%$ of patients after SBRT. McBride et al. (5) found that the mean age of those who experienced a bounce was significantly younger than those who did not. Vu et al. (22) reported that Younger age was the only factor that predicted PSA bounce following SBRT for prostate cancer. However, age was not associated with PSA bounce in our study.

Our study should be examined in the context of study design. Our study is limited by retrospective nature of the analysis and the small number of patients. There were no strict protocols for the clinical decision-making process. Future studies should employ more comprehensive instruments to assess the effect of prostate SBRT.

SBRT using Cyberknife was well tolerated for these patients with localized prostate cancer. Rate of late GI and GU toxicity are comparable to conventional fractionated radiation therapy and brachytherapy. Our 5-year biochemical relapse free survival rate of $93.3 \%$ seems to be favorable.

\section{Acknowledgments}

This work was supported by INHA university research grant.

\section{Footnote}

Conflict of Interest: The authors have no conflicts of interest or financial ties to disclose.

\section{References}

1. Siegel RL, Miller KD, Jemal A. Cancer statistics. CA. 2015;65(1):5-29. doi: 10.3322/caac.21254.

2. Katz AJ, Santoro M, Diblasio F, Ashley R. Stereotactic body radiotherapy for localized prostate cancer: disease control and quality of life at 6 years. Radiat Oncol. 2013;8:118. doi: 10.1186/1748-717X-8-118. [PubMed: 23668632].

3. Chen LN, Suy S, Uhm S, Oermann EK, Ju AW, Chen V, et al. Stereotactic body radiation therapy (SBRT) for clinically localized prostate cancer: the Georgetown University experience. Radiat Oncol. 2013;8:58. doi: 10.1186/1748-717X-8-58. [PubMed: 23497695].

4. King CR, Brooks JD, Gill H, Presti JJ. Long-term outcomes from a prospective trial of stereotactic body radiotherapy for low-risk prostate cancer. Int J Radiat Oncol Biol Phys. 2012;82(2):877-82. doi: 10.1016/j.ijrobp.2010.11.054. [PubMed: 21300474]. 
5. McBride SM, Wong DS, Dombrowski JJ, Harkins B, Tapella P, Hanscom $\mathrm{HN}$, et al. Hypofractionated stereotactic body radiotherapy in lowrisk prostate adenocarcinoma: preliminary results of a multiinstitutional phase 1 feasibility trial. Cancer. 2012;118(15):3681-90. doi: 10.1002/cncr.26699. [PubMed: 22170628].

6. Fowler JF, Ritter MA, Chappell RJ, Brenner DJ. What hypofractionated protocols should be tested for prostate cancer?. Int J Radiat Oncol Biol Phys. 2003;56(4):1093-104. [PubMed: 12829147].

7. Brenner DJ, Hall EJ. Fractionation and protraction for radiotherapy of prostate carcinoma. Int J Radiat Oncol Biol Phys. 1999;43(5):1095-101. [PubMed: 10192361].

8. Brenner DJ, Martinez AA, Edmundson GK, Mitchell C, Thames HD, Armour EP. Direct evidence that prostate tumors show high sensitivity to fractionation (low alpha/beta ratio), similar to late-responding normal tissue. Int J Radiat Oncol Biol Phys. 2002;52(1):6-13. [PubMed: 11777617].

9. Fowler JF. The radiobiology of prostate cancer including new aspects of fractionated radiotherapy.Acta Oncol. 2005;44(3):265-76. doi: 10.1080/02841860410002824. [PubMed:16076699].

10. Martinez AA, Demanes J, Vargas C, Schour L, Ghilezan M, Gustafson GS. High-dose-rate prostate brachytherapy: an excellent acceleratedhypofractionated treatment for favorable prostate cancer. Am J Clin Oncol. 2010;33(5):481-8. doi: 10.1097/COC.0b013e3181b9cd2f. [PubMed: 19952715].

11. Park YH, Choi IY, Yoon SC, Jang HS, Moon HW, Hong SH, et al. Prostatespecific antigen kinetics after primary stereotactic body radiation therapy using CyberKnife for localized prostate cancer. Prostate Int. 2015;3(1):6-9. doi: 10.1016/j.prnil.2015.02.003. [PubMed: 26157760].

12. Abramowitz MC, Li T, Buyyounouski MK, Ross E, Uzzo RG, Pollack A, et al. The Phoenix definition of biochemical failure predicts for overall survival in patients with prostate cancer. Cancer. 2008;112(1):55-60. doi:10.1002/cncr.23139. [PubMed: 17968996].

13. Patel C, Elshaikh MA, Angermeier K, Ulchaker J, Klein EA, Chehade $\mathrm{N}$, et al. PSA bounce predicts early success in patients with permanent iodine-125 prostate implant. Urology. 2004;63(1):110-3. [PubMed: 14751360].

14. Anwar M, Weinberg V, Chang AJ, Hsu IC, Roach M3, Gottschalk A. Hypofractionated SBRT versus conventionally fractionated EBRT for prostate cancer: comparison of PSA slope and nadir. Radiat Oncol. 2014;9:42. doi: 10.1186/1748-717X-9-42. [PubMed: 24484652].
15. Ray ME, Thames HD, Levy LB, Horwitz EM, Kupelian PA, Martinez AA, et al. PSA nadir predicts biochemical and distant failures after external beam radiotherapy for prostate cancer: a multiinstitutional analysis. Int J Radiat Oncol Biol Phys. 2006;64(4):1140-50. doi: 10.1016/j.ijrobp.2005.07.006. [PubMed: 16198506].

16. Arcangeli G, Saracino B, Gomellini S, Petrongari MG, Arcangeli S, Sentinelli S, et al. A prospective phase III randomized trial of hypofractionation versus conventional fractionation in patients with highrisk prostate cancer. Int J Radiat Oncol Biol Phys. 2010;78(1):11-8. doi: 10.1016/j.ijrobp.2009.07.1691. [PubMed: 20047800].

17. Hoskin PJ, Motohashi K, Bownes P, Bryant L, Ostler P. High dose rate brachytherapy in combination with external beam radiotherapy in the radical treatment of prostate cancer: initial results of a randomised phase three trial. Radiother Oncol. 2007;84(2):114-20. doi: 10.1016/j.radonc.2007.04.011. [PubMed:17531335].

18. Martinez A, Gonzalez J, Spencer W, Gustafson G, Kestin L, Kearney D, et al. Conformal high dose rate brachytherapy improves biochemical control and cause specific survival in patients with prostate cancer and poor prognostic factors. J Urol. 2003;169(3):974-9. doi: 10.1097/01.ju.0000052720.62999.a9. [PubMed: 12576825] discussion 979-80.

19. Demanes DJ, Martinez AA, Ghilezan M, Hill DR, Schour L, Brandt D, et al. High-dose-rate monotherapy: safe and effective brachytherapy for patients with localized prostate cancer. Int J Radiat Oncol Biol Phys. 2011;81(5):1286-92. doi: 10.1016/j.ijrobp.2010.10.015. [PubMed: 21310546].

20. Zelefsky MJ, Chan H, Hunt M, Yamada Y, Shippy AM, Amols H. Longterm outcome of high dose intensity modulated radiation therapy for patients with clinically localized prostate cancer. $J$ Urol. 2006;176(4 Pt 1):1415-9. doi: 10.1016/j.juro.2006.06.002. [PubMed: 16952647].

21. Katz AJ, Kang J. Stereotactic body radiotherapy as treatment for organ confined low- and intermediate-risk prostate carcinoma, a 7year study. Front Oncol. 2014;4:240. doi: 10.3389/fonc.2014.00240. [PubMed: 25229051].

22. Vu CC, Haas JA, Katz AE, Witten MR. Prostate-specific antigen bounce following stereotactic body radiation therapy for prostate cancer. Front Oncol. 2014;4:8. doi: 10.3389/fonc.2014.00008. [PubMed: 24478988]. 